Research Article

\title{
A study to evaluate prescribing pattern of antibiotics among patients of urinary tract infection with preexisting renal disorders in a tertiary care hospital
}

\author{
Dinesh K. Dhodi*, Sarita Jaiswar, Sagar B. Bhagat, Rohini S. Gambre
}

\begin{abstract}
Department of Pharmacology, Grant Government Medical College \& Sir JJ Group of Hospitals, Mumbai, Maharashtra, India

Received: 05 June 2014 Accepted: 02 July 2014

\section{*Correspondence to: \\ Dr. Dinesh K. Dhodi, Email: dhodidinesh@gmail. com}

C 2014 Dhodi DK et al. This
is an open-access article
distributed under the terms
of the Creative Commons
Attribution Non-Commercial
License, which permits
unrestricted non-commercial
use, distribution, and
reproduction in any medium,
provided the original work is
properly cited.

(C) 2014 Dhodi DK et al. This is an open-access article distributed under the terms of the Creative Commons Attribution Non-Commercial License, which permits use, distribution, and reproduction in any medium, properly cited.
\end{abstract}

\begin{abstract}
Background: Urinary tract infection (UTI) is one of the most common bacterial infections encountered and treated worldwide. The objective was to determine the incidence, culture-sensitivity status, prescription pattern of antibiotics and response to various antibiotics and other therapeutic considerations in patients of complicated UTI (cUTI).

Methods: The observational prospective analytical study conducted in Department of Nephrology, Grant Government Medical College and Sir JJ Group of Hospitals, Mumbai.

Results: A total percentage of patients having UTI was $13.08 \%$. Of these 200 patients, 119 patients were female $(60 \%)$ and 81 patients were male (41\%). Among male, the prevalence was seen more in geriatric age group and among female in adult group. Symptomatologically, fever was the most common symptom. Among male, diabetes mellitus was most commonly associated with cUTI whereas recurrent UTI were more common among female. Of the 200 patients, culture sensitivity was done in 133 patients. Escherichia coli was found to be the most common organism. Quinolones were the most commonly prescribed first line drug followed by betalactamase inhibitors. Combinations such as cefoperazone + linezolid were the most commonly prescribed second-line drug, followed by combination of ceftriaxone or cefoperazone with metronidazole. Paracetamol was the most common non antibiotic support given.

Conclusions: The results of this study may not be representative of the general population; but UTIs are often treated empirically, and susceptibility tests are often carried out only when the patient has failed one or more courses of antibiotics.
\end{abstract}

Keywords: Antibiotic, Renal disorder, Urinary tract infection

\section{INTRODUCTION}

Urinary tract infection (UTI) is one of the most common bacterial infections encountered and treated worldwide. ${ }^{1}$ It is the second most common cause of bacteremia in hospitalized patients, accounting for $35 \%$ of nosocomial infection. ${ }^{2}$ Worldwide, it is estimated that there are 150 million UTIs per annum, with symptomatic UTI occurring in as many as 7 million, of which 100, 000 requires hospitalizations., The prevalence and incidence of UTI is much more in women than in men, at a ratio of 8:1, due to anatomical and physiological reasons. ${ }^{5,6}$ Malnutrition, poor hygiene, low socioeconomic statuses are associated with UTI, and these factors are rife in rural settings. ${ }^{7}$
UTI is also a major cause of the economic burden in the society, the total cost of UTIs treated with prescription antibiotics is 1.6 billion US $\$ .^{8}$ Most UTIs are uncomplicated cystitis caused by Escherichia coli in otherwise healthy individual. These infections are easily managed with short-term oral antimicrobial therapy. ${ }^{9}$ Complicated UTIs (cUTIs) are frequently associated with either structural or functional urinary tract abnormalities such as calculi (stones), indwelling catheters or other drainage devices, obstruction, immunosuppression, renal failure, renal transplantation, and pregnancy..$^{10}$ Treatment of cUTI are often less successful than treatment of uncomplicated UTI. ${ }^{9}$ The reasons for this include the underlying abnormalities, particularly obstruction, which 
may prevent organism eradication; the tendency for recurrent infections; and the presence of organisms with increasing levels of resistance to antimicrobials agents after their repeated course..$^{9,11}$

The bacterial spectrum of cUTI is heterogeneous and comprises a wide range of Gram-negative and Gram-positive species. These bacterial spectrums can vary from place to place and even among patients of the same institution. ${ }^{12}$ In general, Gram-negative species account for approximately $70-80 \%$ of the spectrum and comprise of $E$. coli, followed by Klebsiella, Pseudomonas, Proteus, Enterobacter and Citrobacter. The Gram-positive pathogens account for about $15-30 \%$ of the spectrum and comprise of enterococci, followed by staphylococci. ${ }^{13-17}$

It is well-recognized that, over the last several decades, antimicrobial resistance has evolved in uropathogens responsible for cUTIs. Not unexpectedly, patients who experience a cUTI are more likely to harbor multiresistant pathogens than patients with acute uncomplicated UTIs. ${ }^{17-19}$ Furthermore, the increase in rates of antibiotic resistance among Enterobacteriaceae has posed challenges in choosing empiric regimens, especially when an infections due to multidrug-resistant Enterobacteriaceae, extendedspectrum beta-lactamases has been reported worldwide. ${ }^{19}$ In addition, other mechanisms of resistance among urinary pathogens including Klebsiella pneumoniae carbapenemases and New Delhi metallo-beta-lactamases have also been increasingly described. ${ }^{19,20}$

Recent guidelines from the infectious diseases society recommended that empiric antibiotic therapy for UTIs should be based on local resistance data, drug availability, and antibiotic intolerance/allergy history of treated patients. ${ }^{21,22}$ However, current policies are based largely on ecological relationships between prescribing and resistance, and there are only limited data at the level of the individual patient. ${ }^{23,24}$ Interventions to improve the quality of antibiotic prescribing could be enhanced if antibiotic policy were based individually for every patient based on safety, tolerability and resistance pattern. ${ }^{25}$ This study was designed for gaining knowledge about the type of pathogens responsible for cUTIs and their susceptibility patterns which may help the clinicians to choose the right empirical treatment. Therefore, it was aimed to determine the incidence, culture-sensitivity status, prescription pattern of antibiotics and response to various antibiotics and other therapeutic considerations in patients of cUTI.

\section{METHODS}

This was an observational prospective analytical study conducted in outpatient and inpatient department of Nephrology, Grant Medical College and Sir JJ groups of Hospitals, Mumbai, after obtaining Institutional Ethics Committee clearance. The study was conducted for a period of six months, from January 2011 to June 2011, during which 200 prescriptions were analyzed. The sample size selection was based on the WHO guidelines for conducting drug utilization studies and on the basis of other such studies conducted in the past. Patients were classified as having cUTI based on the criteria defined by Rubenstein and Shaeffer. ${ }^{26}$ This study included patients of all age and sex having UTI with a pre-existing renal disorder from the Nephrology outpatient, inpatient department or referred from other departments of Sir JJ Groups of Hospitals. Patients with immunocompromised condition or those on immunosuppressant drugs and patients with emergency life threatening conditions or any other infection besides UTI were excluded from the study.

\section{RESULTS}

In a six-month study, from January 2011 to June 2011, a total of 1529 patients attended Nephrology Department, out of which 1406 were from the Nephrology outpatient department and 123 patients were from the IPD and referred patients. With having sample size of 200, total percentages of patients having UTI is $13.08 \%$ (Figure 1). Out of these 200 patients, 119 patients were female $(60 \%)$, and 81 patients were male (41\%) (Figure 2). The patients were of all age group from pediatric to geriatric. Among the male, the prevalence was seen more in geriatric age group and amongst female prevalence was seen more in adult group (Table 1). Symptomatologically, fever was the most common symptom followed by dysuria and urgency (Figure 3). Among the male, Diabetes mellitus was the most common factor associated with cUTI whereas recurrent UTI was the more common among female (Figures 4 and 5). Of the 200 patients, culture sensitivity was done in 133 patients considering patients' severity of infection and extent of renal disease involvement (Figure 6). E. coli was found to be the most common organism accounting for about $65 \%$ followed by Staphylococcus aureus, Pseudomonas, Klebsiella, Candida, Proteus. In some of the patients, there were mixed infections involving E. coli with enterococci or Klebsiella or Candida. Total number of patients infected with a particular organism has been depicted in the table given below (Figure 7). Quinolones such as ciprofloxacin, levofloxacin were the most commonly prescribed first line drug followed by betalactamase inhibitors such as penicillin and cephalosporin

Table 1: Age distribution of UTI in male and female.

\begin{tabular}{|lcc|}
\hline Age group (years) & Males (\%) & Females $(\%)$ \\
\hline $0-15$ & $9(11.11)$ & $7(8.6)$ \\
\hline $16-30$ & $6(7.40)$ & $19(23.45)$ \\
\hline $31-45$ & $16(19.75)$ & $39(48.14)$ \\
\hline $46-60$ & $21(25.92)$ & $32(39.50)$ \\
\hline$>60$ & $29(35.80)$ & $22(27.16)$ \\
\hline
\end{tabular}

UTI: Urinary tract infection 
(Figure 8). Combinations such as cefoperazone + linezolid were the most commonly prescribed second-line drug followed by combination of ceftriaxone or cefoperazone with metronidazole (Figure 9). Paracetamol was the most common non antibiotic support given (Figure 10).



Figure 1: Distribution of urinary tract infection.

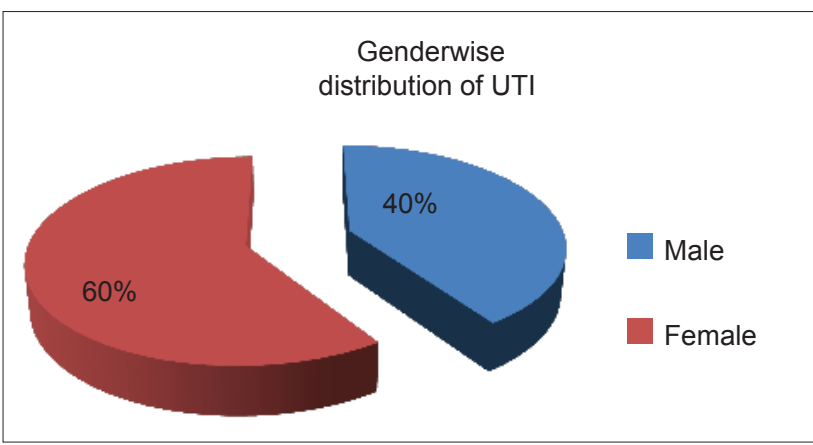

Figure 2: Gender wise distribution of urinary tract infection.

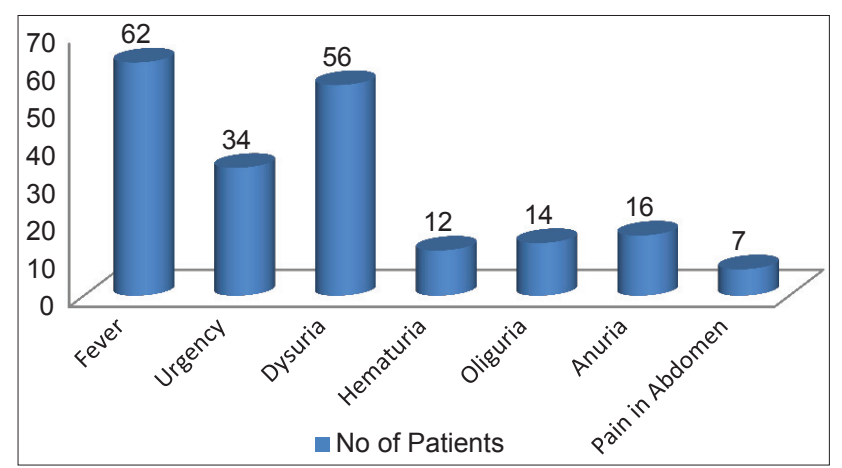

Figure 3: Symptoms in patients with urinary tract infection.



Figure 4: Risk factors for complicated urinary tract infection in males.

\section{DISCUSSION}

Our study observes that the prevalence of cUTI was high among females $(60 \%)$ than males $(41 \%)$ which was in contrast to the study conducted in South India by Mahesh et al. $2010^{27}$ were prevalence was seen more in males $(63 \%)$ than in females (37\%) and by Arul Prakasam et al. $2012^{28}$ where prevalence in male was $85.7 \%$. In our study, among females highest prevalence is seen in age group of 31-45

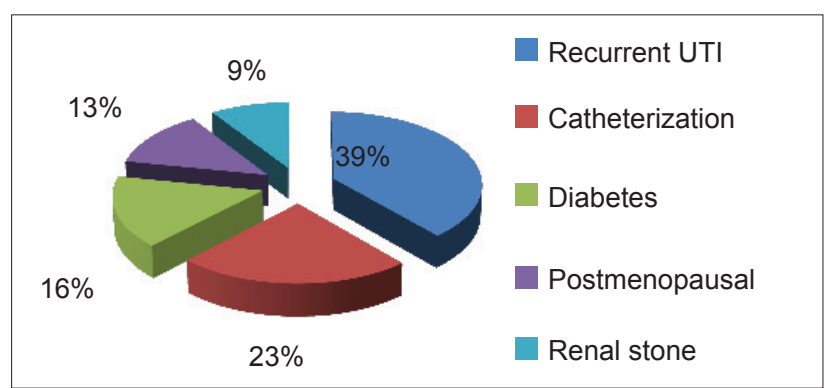

Figure 5: Risk factors for complicated urinary tract in females.

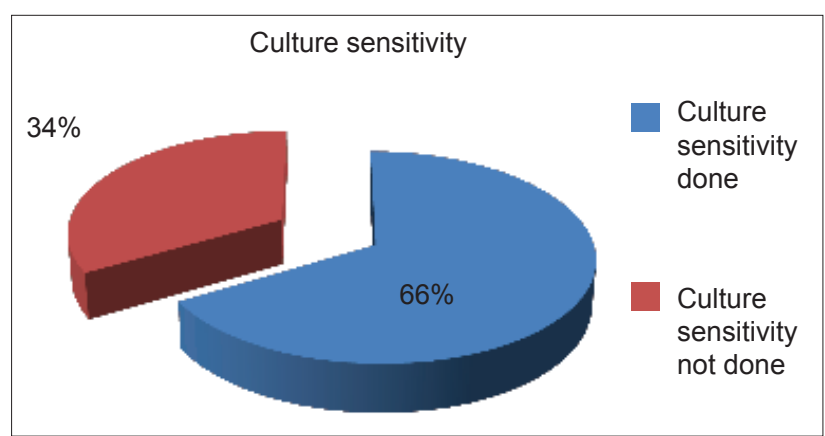

Figure 6: Culture sensitivity report.

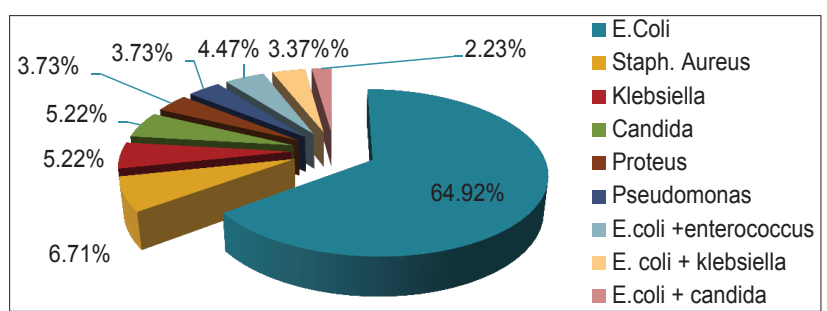

Figure 7: Organism causing urinary tract infection.

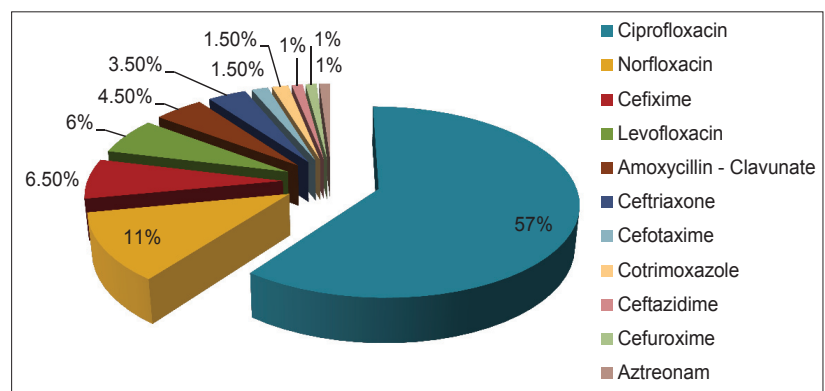

Figure 8: First-line antibiotic prescribe to patients of urinary tract infection. 


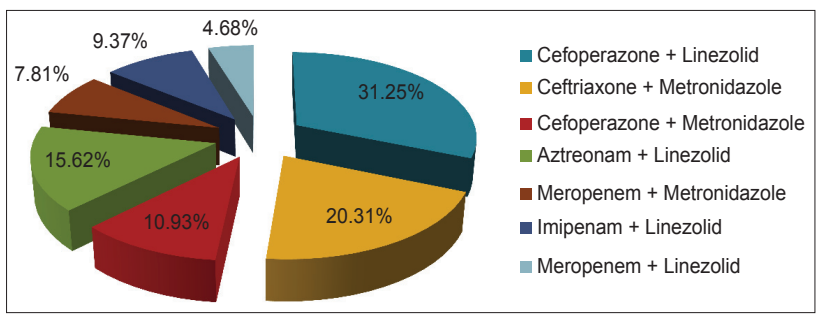

Figure 9: Second-line antibiotics prescribe to patients of urinary tract infection.

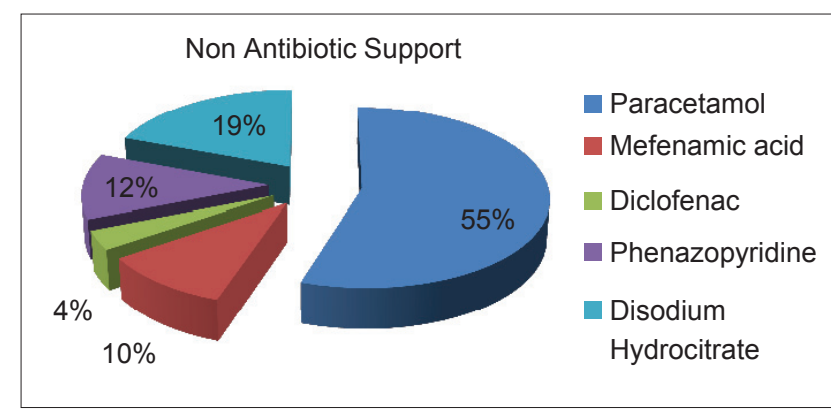

Figure 10: Nonantibiotic support.

(48.14\%) followed by 46-60 age group (39.50\%). Close proximity of female urethral meatus to anus, shorter urethra and sexual intercourse have been reported as factors that influences this higher prevalence in women. Among males an increased prevalence of UTI was recorded among the elderly age group, $>60(35.80 \%)$, and $41-50(25.92 \%)$ than among young age patients $(7.40 \%)$. Increasing frequency of prostate disease in males and diabetes mellitus are responsible for increasing the incidence of UTI in elderly patients. ${ }^{29}$ Fever and Dysuria were the most common symptoms as with similar studies worldwide. Among male, Diabetes mellitus $(38 \%)$ was the most common factor responsible for cUTI followed by smoking (24\%) and benign prostatic hyperplasia $(17 \%)$ and least with renal stone (7\%). This was in contrast to study conducted Arul Prakasam et al. $2012^{28}$ were smoking was found to be the most common factor followed by Diabetes. However, our results were in agreement with other study which showed that UTI is more common in diabetic male patients. ${ }^{27}$ Among females, recurrent UTI was the most common factor (39\%) followed by catheterization (23\%) and diabetes (17\%). This was in agreement with the study conducted in the past. ${ }^{28}$ Among all, who were sent for culture sensitivity, E. coli $(64.92 \%)$ topped the list of organisms causing cUTI which was similar to studies conducted worldwide such as by Arslan et al.,${ }^{30}$ Chen et al., ${ }^{31}$ Peterson et al. ${ }^{32}$ also by Nicolle. ${ }^{33}$ In our study, the other organisms isolated from culture were $S$. aureus $(6.71 \%)$, Pseudomonas $(3.73 \%)$, Klebsiella $(5.22 \%)$. All the cUTI suspects were initially treated with empirical antibiotics. Quinolones were the most widely prescribed antibiotics for the empirical therapy of cUTI followed by cephalosporin, penicillin and cotrimoxazole which was similar to studies done in the past in India. ${ }^{28}$ Among fluoroquinolones, ciprofloxacin was the most preferred, followed by norfloxacin and levofloxacin and least was ofloxacin. Second line antibiotic were started on those who did not show any good result with the first line antibiotic, combination of cefoperazone and linezolid was the most preferred second line antibiotic. Intravenous route gave the entire second line antibiotic.

\section{CONCLUSION}

The results of this study may not be representative of the general population; but UTIs are often treated empirically, and susceptibility tests are often carried out only when the patient has failed one or more courses of antibiotics. These data may be used to determine trends in antimicrobial susceptibilities, to formulate local antibiotic policies, to compare local with national data and overall to assist clinicians in the rational choice of antibiotic therapy to prevent misuse, or overuse, of antibiotics. As lesser new antibiotics are available for their management, we need to be concerned of this issue in years to come especially in tertiary care centers. A unified antibiotic protocol is necessary to limit this increase and reduce the squeal of cUTI.

\section{Funding: No funding sources}

Conflict of interest: None declared

Ethical approval: The study was approved by the Institutional Ethics Committee

\section{REFERENCES}

1. Sibi G, Aheibam PD, Fouzia K, Patil BR. Prevalence microbiologic profile of urinary tract infection and its treatment with trimethoprim in diabetic patients. Res $\mathrm{J}$ Microbiol. 2011;6(6):543-51.

2. Wasnik DD, Tumane PM. Prevalence and antibacterial susceptibility pattern of urinary tract infection causing human pathogenic bacteria. Asian J Biomed Pharm Sci. 2012;2(15):1-3.

3. Sussman M. In: Hausler WJ Jr, Sussman M editors. Topley and Wilson's Microbiology and Microbial Infections. 9th Edition. London: Arnold; 1998: 601-21.

4. Stamm WE, Norrby SR. Urinary tract infections: disease panorama and challenges. J Infect Dis. 2001;183 Suppl 1:S1-4.

5. Patel S, Taviad PP, Sinha M, Javadekar TB, Chaudhari VP. Urinary tract infections (UTI) among patients At G.G. Hospital \& Medical College, Jamnagar. Natl J Community Med. 2012;3(1):138-45.

6. Akhtar N. Urinary tract bacterial pathogens their antimicrobial susceptibility patterns at Bahawalpur. Professional. 2000;7(2):131-7.

7. Ahmed AB, Ghadeer AS. Recurrent urinary tract infections management in women a review. Sultan Qaboos Univ Med J. 2013;13(3):359-67.

8. Maripandi A, Ali A, Salamah A, Amuthan M. Prevalence and antibiotics susceptibility of uropathogens in patients from a rural environment, Tamilnadu. Am J Infect Dis. 2010;6(2):29-33.

9. Bankole H O, Omoregie R, Olley M, Joshua A. Urinary tract infection in a rural community of Nigeria. N Am J Med Sci. 2011;3(2):75-7.

10. Rahn DD. Urinary tract infections: contemporary management. Urol Nurs. 2008;28(5):333-41. 
11. Foxman B, Barlow R, D’Arcy H, Gillespie B, Sobel JD. Urinary tract infection: self-reported incidence and associated costs. Ann Epidemiol. 2000;10(8):509-15.

12. Foxman B. Epidemiology of urinary tract infections: incidence, morbidity, and economic costs. Am J Med. 2002;113 Suppl 1A:5S-13.

13. Mathai D, Jones RN, Pfaller MA, SENTRY Participant Group North America. Epidemiology and frequency of resistance among pathogens causing urinary tract infections in 1,510 hospitalized patients: a report from the SENTRY antimicrobial surveillance program (North America). Diagn Microbiol Infect Dis. 2001;40:129-36.

14. Bryan CS, Reynolds KL. Community-acquired bacteremic urinary tract infection: epidemiology and outcome. J Urol. 1984;132(3):490-3.

15. Kahlmeter G, ECO.SENS. An international survey of the antimicrobial susceptibility of pathogens from uncomplicated urinary tract infections: the ECO.SENS Project. J Antimicrob Chemother. 2003;51(1):69-76.

16. Shanthi J, Kayathri S. Incidence, distribution and antibiogram of uropathogens isolated from patients with urinary tract infections. Adv Appl Sci Res. 2012;3(6):3410-4.

17. Sanchez GV, Master RN, Karlowsky JA, Bordon JM. In vitro antimicrobial resistance of urinary Escherichia coli isolates among U.S. outpatients from 2000 to 2010. Antimicrob Agents Chemother. 2012;56(4):2181-3.

18. Qi C, Pilla V, Yu JH, Reed K. Changing prevalence of Escherichia coli with CTX-M-type extended-spectrum betalactamases in outpatient urinary E. coli between 2003 and 2008. Diagn Microbiol Infect Dis. 2010;67(1):87-91.

19. Gupta N, Limbago BM, Patel JB, Kallen AJ. Carbapenemresistant Enterobacteriaceae: epidemiology and prevention. Clin Infect Dis. 2011;53(1):60-7.

20. Kumarasamy KK, Toleman MA, Walsh TR, Bagaria J, Butt F, Balakrishnan R, et al. Emergence of a new antibiotic resistance mechanism in India, Pakistan, and the UK: a molecular, biological, and epidemiological study. Lancet Infect Dis. 2010;10(9):597-602.

21. Gupta K, Hooton TM, Naber KG, Wullt B, Colgan R, Miller LG, et al. International clinical practice guidelines for the treatment of acute uncomplicated cystitis and pyelonephritis in women: a 2010 update by the Infectious Diseases Society of America and the European Society for Microbiology and Infectious Diseases. Clin Infect Dis. 2011;52(5):e103-20.

22. Hooton TM, Bradley SF, Cardenas DD, Colgan R, Geerlings SE, Rice JC, et al. Diagnosis, prevention, and treatment of catheter-associated urinary tract infection in adults: 2009 International Clinical Practice Guidelines from the Infectious Diseases Society of America. Clin Infect Dis. 2010;50(5):625-63.
23. Hillier SL, Magee JT, Howard AJ, Palmer SR. How strong is the evidence that antibiotic use is a risk factor for antibioticresistant, community-acquired urinary tract infection? J Antimicrob Chemother. 2002;50(2):241-7.

24. Steinke D, Davey P. Association between antibiotic resistance and community prescribing: a critical review of bias and confounding in published studies. Clin Infect Dis. 2001;33 Suppl 3:S193-205.

25. Hillier S, Roberts Z, Dunstan F, Butler C, Howard A, Palmer S. Prior antibiotics and risk of antibiotic-resistant community-acquired urinary tract infection: a case-control study. J Antimicrob Chemother. 2007;60(1):92-9.

26. Rubenstein JN, Schaeffer AJ. Managing complicated urinary tract infections: the urologic view. Infect Dis Clin North Am. 2003;17(2):333-51.

27. Pargavi B, Mekala T, Thamarai Selvi A, Moorthy K. Prevalence of urinary tract infection among diabetics patients in Vandavasi, Tamilnadu, India. Int J Biol Technol. 2011;2(2):42-5

28. Arul Prakasam KC, Dileesh Kumar KG, Vijayan M. A cross sectional study on distribution of urinary tract infection and their antibiotic utilizations pattern in Kerala. Int J PharmTech Res. 2012;4(3):1309-16.

29. Mahesh E, Ramesh D, Indumathi VA, Punith K, Raj K, Anupama HA. Complicated urinary tract infection in a tertiary care centre in South India. Al ameen J Med Sci. 2010;3(2):120-7.

30. Arslan H, Azap OK, Ergönül O, Timurkaynak F, Urinary Tract Infection Study Group. Risk factors for ciprofloxacin resistance among Escherichia coli strains isolated from community-acquired urinary tract infections in Turkey. J Antimicrob Chemother. 2005;56(5):914-8.

31. Chen SS, Chen KK, Lin AT, Chang YH, Wu HH, Hsu TH, et al. Complicated urinary tract infection: analysis of 179 patients. Zhonghua Yi Xue Za Zhi (Taipei). 1998;61(11):651-6.

32. Peterson J, Kaul S, Khashab M, Fisher A, Kahn JB. Identification and pretherapy susceptibility of pathogens in patients with complicated urinary tract infection or acute pyelonephritis enrolled in a clinical study in the United States from November 2004 through April 2006. Clin Ther. 2007;29(10):2215-21.

33. Nicolle LE. A practical guide to the management of complicated urinary tract infection. Drugs. 1997;53(4):583-92.

doi: $10.5455 / 2319-2003 . i j b c p 20140825$

Cite this article as: Dhodi DK, Jaiswar S, Bhagat SB,

Gambre RS. A study to evaluate prescribing pattern of antibiotics among patients of urinary tract infection with preexisting renal disorders in a tertiary care hospital. Int $\mathbf{J}$ Basic Clin Pharmacol 2014;3:687-91. 